\title{
Dispositivo de intervención y gubernamentalidad del sistema proteccional de la infancia: discursos de trabajadores y trabajadoras de programas de familias de acogida especializadas de la Región de Valparaíso, Chile ${ }^{1}$
}

\author{
Rodrigo Cortés Mancilla²
}

\section{RESUMEN}

Chile protege a la infancia en condición de vulneración de sus derechos, mediante una institucionalidad gubernamental específica. El Estado pone en funcionamiento una red de dispositivos sobre el cuerpo de niños, niñas y adolescentes en protección, que configuran subjetividades y relaciones de poder construidas y articuladas en diferentes programas o proyectos. En el país, la pesquisa de alternativas a programas de residencia ha implicado el financiamiento de organizaciones no gubernamentales por parte del Estado, lo que ha posibilitado establecer ambientes alternativos relativamente protectores y estables. En esta cotidianidad circulan y se entrelazan sujetos, relaciones de poder/saber, mecanismos regulatorios y marcos referenciales. Uno de ellos es el denominado "Programa de Familias de Acogida Especializadas". Este estudio, llevado a cabo mediante entrevistas en profundidad, se focaliza en el discurso de trabajadores y trabajadoras de dicho programa, en vistas a dilucidar mecanismos, relaciones y configuraciones que se establecen en el desarrollo de esta tarea, y que sirven de soporte a la protección de una infancia vulnerada en sus derechos.

Palabras clave: infancia, gubernamentalidad, dispositivo, relaciones, sujeto

Artículo recibido 15/11/2018. Artículo aprobado 25/12/2108.

Chileno. Académico, Escuela de Trabajo Social, Facultad de Humanidades y Ciencias Sociales, Universidad Andrés Bello. E-mail: rcortes@unab.cl. En la elaboración de este artículo participaron como coautores los siguientes egresados de la carrera de Trabajo Social de la Universidad Andrés Bello: Catalina Urrutia Ruz, Javiera Subiabre Villegas y Yerenni Flores Lizardi. 


\title{
Intervention Programs and Governance of the Chile Protection System: Workers' Discourse Within Specialized Foster Family Programs in the Region of Valparaiso, Chile
}

\author{
ABSTRACT
}

Chile has a special governmental system to protect vulnerable children from the infringement of their rights. The Government operates a network of programs for all children and youth under protection, which configure subjectivities and power relations constructed and coordinated among different programs or projects. In the country, the study of alternatives to orphanage programs has implied State financing for Non-Governmental Organizations, thus enabling the establishment of relatively protective and stable alternative environments. This system is characterized by the circulation and intertwining of subjects, power/knowledge relationships, regulatory mechanisms and frameworks of reference. One of these is the Specialized Foster Family Program. This study, carried out through in-depth interviews, is focused on the discourse of this program's workers in order to shed light on the mechanisms, relations and configurations established in the development of their intervention work which serves as a support for the protection of at-risk children.

Palabras clave: childhood, governance, program, relations, subject

\section{dispositivo de intervenção e governamentalidade do sistema protecional da infância: discursos de trabalhadores e trabalhadoras de programas de famílias de acolhimento especializadas da Região de Valparaíso, Chile}

\section{RESUMO}

O Chile protege a infância em condição de vulneração de Direitos através de una institucionalidade governamental específica. O Estado põe em funcionamento uma rede de dispositivos sobre o corpo de crianças e adolescentes em proteção, que configuram subjetividades e relações de poder construídas e articuladas em diferentes programas ou projetos. No país, a pesquisa de alternativas a programas de residência tem implicado o financiamento de Organizações Não Governamentais por parte do Estado, o que tem possibilitado estabelecer ambientes alternativos relativamente protetores e estáveis. Nesta cotidianidade circulam e se entrelaçam sujeitos, relações de poder/saber, mecanismos regulatórios e marcos referenciais. Um deles é o denominado Programa de Famílias de Acolhimento Especializadas. Este estudo, efetuado através de entrevistas em profundidade, foca-se no discurso de trabalhadores e trabalhadoras de dito programa, em vista a definir mecanismos, relações e configurações que se estabelecem no 
desenvolvimento desta tarefa e que servem de suporte à proteção de uma infância vulnerada em seus direitos.

Palavras-chave: infância, governamentalidade, dispositivo, relações, sujeito

\section{Introducción}

Este artículo es una síntesis de la investigación que indagó en los discursos referidos a procesos de intervención social de un programa que trabaja con infancia "en situación de vulneración de sus Derechos", dispositivo implementado por el Servicio Nacional de Menores $^{3}$ (desde ahora SENAME) de la Región de Valparaíso, en Chile. Este estudio se enfocó en las prácticas discursivas de los trabajadores y las trabajadoras de un programa particular denominado "Programa de Familia de Acogida Especializada" (desde ahora FAE), en sus relaciones con niños, niñas y adolescentes (en adelante NNA) y sus contextos familiares, que configuran parte de la dimensión más cotidiana de la política de protección de la infancia desarrollada por el Estado de Chile.

El Programa FAE se configura en la política nacional de la infancia de Chile, con dependencia administrativa y técnica en el SENAME, y tiene como objetivo principal: asegurar una atención de calidad bajo condiciones fundamentales de protección, afecto, contención $y$ desarrollo en una familia de acogida, a NNA que han debido ser separados de su medio familiar mientras se restablece su derecho a vivir en una situación familiar estable (SENAME, 2010, p. 15).

El FAE, en sus inicios, se denominó "Apoyo Familiar o Ayuda Intrafamiliar", posteriormente "Colocaciones Familiares", todas modalidades que aspiraban a mantener a los niños/as con su familia (generalmente con la madre u otros parientes), otorgándoles un soporte económico mientras se resolvían diversas situaciones de vulnerabilidad, evitando así su internación en centros residenciales” (Martínez, 2011, p. 6). Así, las familias de acogida son gru-

Organismo gubernamental centralizado, colaborador del sistema judicial y dependiente del Ministerio de Justicia, encargado de la protección de derechos de niños, niñas y adolescentes. 
pos familiares con relaciones alternativas y transitorias, que se encuentran en disposición de ser evaluadas, de generar una red de cuidado, de satisfacción de necesidades, de apoyo, entre otros criterios que determina el SENAME. Estas familias pueden ser las que poseen un vínculo consanguíneo con el NNA, denominadas "familias extensas" y, por otra parte, están las familias que no tienen vínculo consanguíneo (Martinez, 2011).

En este artículo se describen los ejes teórico-conceptuales del estudio, para posteriormente describir aspectos de la estrategia metodológica empleada, continuando con los hallazgos de acuerdo con tres niveles de análisis desarrollados, cerrando con apreciaciones finales.

\section{De la gubernamentalidad liberal chilena y la infancia}

La configuración de la política y la institucionalidad de la infancia en Chile se consolida en el año 1989, momento en que el Estado se hace parte de la Convención de los Derechos del Niño, lo que proyectó las posibilidades de reconfiguración y resignificación de políticas, programas y proyectos en cuanto a enfoque, propósitos y estrategias. No obstante, la experiencia ha sido más compleja de lo que se preveía, ya que el Estado Chileno y sus estructuras estaban sustentadas en una superestructura neoliberal, en la que la política pública y económica ha estado marcada por esa corriente ideológica.

El Estado plantea un relato de rearticulación de los lineamientos para comprender e intervenir en el fenómeno de la infancia, pero, como se planteó, "en la medida de lo posible"4, con ello se mantuvo y reprodujo ese

conjunto constituido por las instituciones, los procedimientos, análisis y reflexiones, los cálculos y las tácticas que permiten ejercer esa forma bien específica, aunque muy comple-

\footnotetext{
Referencia al discurso estatal significado desde la frase de Patricio Aylwin, frente a los juicios de DDHH: "justicia en la medida de lo posible".
} 
ja, de poder que tiene por blanco principal la población, por forma mayor de saber la economía política y por instrumento técnico esencial los dispositivos de seguridad (Foucault, 2001, p. 195).

Con ello se reprodujo esa articulación, esa gubernamentalidad que la dictadura había legado y que los gobiernos de la Concertación de Partidos por la Democracia reprodujeron en estructura y funcionamiento, incluso agudizando la intervención terciaria o privada en el fenómeno (Gruninger 2003; Bresser y Cunill 1998; De la Maza 1997, 2004; Sánchez y Villarroel, 2017). Así, el Estado chileno externalizó y traspasó financiamiento a privados, subsidiándolos significativamente. Crear condiciones favorables para el sector no lucrativo supondría una prioridad política en la agenda gubernamental y una comprensión más clara de las funciones que le caben en el desarrollo social; sin embargo, se lo ha concebido principalmente como parte de un conjunto de agentes privados que cooperan con la función estatal en infancia, a un menor costo. De este modo, el ejercicio de la gubernamentalidad neoliberal en infancia fue tendencia y línea de fuerza hacia la preeminencia de este tipo de poder que se podría llamar "gobierno" sobre todos los NNA y las familias focalizadas por la política, manteniendo "soberanía, disciplina; lo que ha comportado, por una parte, el desarrollo de toda serie de saberes" (Foucault, 2001, p. 195), en las líneas programáticas hacia la infancia.

Las medidas estatales también han operado sobre la producción de conocimientos e intervenciones, en cuanto a la infancia /tutelada/protegida, de cuyo proceso surgen un conjunto de instituciones como organismos colaboradores de SENAME, expertos y perspectivas desde distintas disciplinas, destinadas a constituir un régimen social normalizador (Oyarzún y otros, 2008). Esto tiene como consecuencia una red de dispositivos que intervienen en la vida de niños y niñas que se encuentran en situación y condición de vulnerabilidad. Siendo una de esas respuestas de Estado los programas FAE. 
Con ello, la gubernamentalidad chilena ha implicado una vinculación entre el Estado y la implementación de diversos mecanismos, que se conforman desde dos modelos: la anatomopolítica y la biopolítica (Foucault, 1999, 2001, 2007; Butler 2008; Agamben 2002), los que constituyen dos modalidades de una forma de poder que se organiza en torno a la vida de NNA, de sus familias y de quienes estén vinculados o vinculadas en la implementación de procedimientos o tácticas de Estado, por tanto un biopoder que "aparece como una dinámica regulatoria que apunta a la administración de la vida de ese nuevo sujeto colectivo que es la población, buscando gestionar su potencia vital para hacerla más productiva, más eficiente, más segura, más regular” (Botticelli, 2015, p. 86).

Historicamente, la infancia ha estado sujeta a la anatomopolítica estatal (Salazar 2002, 2003, 2006; Rojas, 2010; Torres 2008; Aguilar, 2008), la que centró su mirada en los cuerpo de niños y niñas de sectores populares, intentando mecanizar esos cuerpos a través de

su educación, el aumento de sus aptitudes, el arrancamiento de sus fuerzas, el crecimiento paralelo de su utilidad y su docilidad, su integración en sistemas de control eficaces y económicos, todo ello quedó asegurado por procedimientos de poder característicos de las disciplinas (Foucault, 1999, p. 83).

Ellos como un proceso de reproducción de logicas de cuidado, disciplinamiento de la infancia desde la colonia. El Estado de Chile ha generado leyes y políticas a través de su institucionalidad, la primera de ellas fue la Ley de protección a la infancia desvalida (1912), promulgada para resolver el tema del abandono paterno, el abuso de menores y algunas formas de explotación, y pese a que su aplicación fue muy discreta, marcó el inicio de una política estatal orientada hacia los niños en "riesgo social" o en "condición de protección".

Por otro parte, la actual política de infancia tiene un legado de diversos programas construidos hacia los NNA en Chile (mortalidad infantil, gota de leche, entre otros), centrados en sus cuerpos, consumidos 
por la mecánica de lo viviente y que sirve de soporte a los procesos biológicos: la proliferación, los nacimientos y la mortalidad, el nivel de salud, la duración de la vida y la longevidad, con todas las condiciones que pueden hacerlos variar; todos esos problemas los toma a su cargo una serie de intervenciones y controles reguladores: una biopolítica de la población (Foucault, 1991, p. 83).

Se entiende la biopolítica como ese "conjunto de cálculos y tácticas que se desarrollan para intervenir sobre la vida colectiva" (Botticelli, 2015, p. 88), siendo una de las tácticas las acciones generadas por programas como el de Familias de Acogida Especializada, que se realiza por medio de marcos regulatorios diversos (Ley de Menores, Ley de Tribunales de Familia, Ley de subvenciones, Orientaciones técnicas, misiones institucionales, entre otros). De esta manera, las intervenciones con NNA con medidas de protección sometidos a la protección del SENAME, se plantean como problema político, así también "como problema a la vez científico, como problema biológico y problema de poder" (Foucault, 2000, p. 222).

Pensar la gubernamentalidad neoliberal chilena en referencia a sus mecanismos, implica comprender al FAE como un dispositivo el que concurren prácticas discursivas vinculadas por mecanismos que configuran la sociedad. Se comprendió al programa FAE, por tanto, como "un conjunto heterogéneo, que incluye discursos, instituciones, reglamentos, leyes, medidas administrativas, enunciados científicos, proposiciones filosóficas, morales" (Agamben, 2015, p. 25), y las relaciones que implican este entramado de "instituciones, sistemas de normas, formas de comportamiento, procesos económicos, sociales, técnicos y tipos de clasificaciones de sujetos, objetos y relaciones entre estos, un juego de relaciones discursivas y no discursivas, de regularidades que rigen una dispersión cuyo soporte son prácticas” (Agamben, 2015, p. 32).

En toda formación social, como los FAE, se producen y reproducen prácticas que condicionan a los sujetos -por ejemplo, el marco normativo impuesto por diversas instituciones-, además de condicionar las relaciones de saber/poder, saber (disciplinario- 
profesional) como instrumento de poder y uno de los elementos más fuertes de dominación. Así el saber y el poder son dos caras de la misma moneda: todo poder genera saber y todo saber proviene de un poder, se implican mutuamente, no son independientes, ya que el poder produce saber y el saber produce y mantiene el poder, y también legitima su ejercicio (Ovejero y Pastor, 2001). Esas relaciones implican un "conjunto de acciones que tienen por objeto otras acciones posibles; operan sobre un campo de posibilidades: inducen, apartan, facilitan, dificultan, extienden, limitan, impiden" (Foucault, 1994, p.146).

Así, el supuesto es que quienes tienen posición de sujeción en el dispositivo estudiado son los trabajadores y las trabajadoras del sistema, asumiendo a la vez que ellos y ellas también están condicionados por otras relaciones. Estos son quienes ejercen poder disciplinario en y sobre la vida cotidiana de los otros. Clasificando a los otros así, "los designa por su individualidad propia, los liga a su identidad, les impone una ley, que se ven obligados a reconocer y que los otros tienen que reconocer en ellos, es una forma de poder que transforma a los individuos en sujetos" (Foucault, 1989, p. 17).

Es por esta posición en la relación que nos aproximamos al sujeto trabajador/trabajadora desde su discurso "como una especie de reserva a los modelos de la visibilidad y sirve espontáneamente de articulación entre lo que se puede ver y lo que se puede decir" (Foucault, 1968, pp. 135-136) del FAE. Indagando desde su discurso en las dimensiones del dispositivo (relaciones, sujetos, mecanismos) de intervención social, denominado Programa FAE, de la Región de Valparaíso.

\section{De la estrategia metodológica}

En la investigación se optó por un enfoque cualitativo, que permitió una aproximación comprensiva e interpretativa crítica de los discursos de los trabajadores del Programa FAE, para comprender e interpretar el fenómeno en términos de su significado. Por 
ello se postuló que la realidad se construye subjetivamente desde quienes participan de la investigación, en tanto sujetos que contribuyen a producir y reproducir el contexto de interacción que se investigó (Sautu, 2005).

Como táctica de la estrategia metodológica se utilizó la entrevista en profundidad, para recoger los discursos desde la experiencia de los y las hablantes, relatos producidos por los entrevistados y recibidos en el acto de escucha e interpretación. Así, en la acción interpretativa, el primer paso fue oír los relatos de campo de trabajadores y trabajadoras como narrativas en perspectiva y no como informaciones, mucho menos como verdades. De este modo, las entrevistas fueron comprendidas como

una interpretación de la realidad realizada, primero, por el narrador bajo la influencia del investigador. La narrativa es, por lo tanto, una versión posible de la historia o del hecho bajo la perspectiva del entrevistado que ya la verbaliza de forma interpretada en su habla o en sus silencios (De Souza Minayo, 2010, pp. 258-259).

La labor interpretativa fue autónoma y vinculada, local y abarcativa, comprensiva y crítica. Como indica Gadamer (1993), se procuró entender al interlocutor/a mejor de lo que él mismo lo hubiera hecho, intentando develar lo que quedó inconsciente u obscuro para él.

Los y las interlocutoras fueron diez trabajadores y trabajadoras de programas de familias de acogida especializada de la Región de Valparaíso, pertenecientes a organismos colaboradores de SENAME. Esta decisión tuvo relación con la factibilidad de acceso a ellos y ellas, además de que cada uno/a contaba con más de un año de experiencia en el Programa, siendo trabajadores de gestión y profesionales de intervención directa.

De acuerdo con lo planteado, se presentarán algunos hallazgos desde los discursos de los y las trabajadores/as, que dan cuenta de tres niveles interpretativos: el primero, con un sentido inductivo, el segundo deductivo y el tercero una relación inductiva-deductiva. 


\section{Deconstruyendo el dispositivo desde categorías emergentes y dimensiones sustantivas}

En el presente apartado, se informa de algunos de los hallazgos de la investigación, dando cuenta de tres niveles de análisis de los discursos de los trabajadores del dispositivo FAE, de la Región de Valparaíso.

Los primeros resultados resultan de un movimiento inductivo, presentando dos categorías que se construyeron desde la interpretación de los discursos de los y las entrevistados/as; el segundo momento será de orden deductivo, en el que se vincularon los discursos de las y los entrevistadas/os con las dimensiones de la teoría sustantiva desarrollada. El tercer nivel, una articulación entre los dos movimientos planteados.

\section{Categorías emergentes desde el discurso de los y las trabajadores/as}

La imagen 1 presenta las cuatro categorías emergentes desde el discurso de los entrevistados. Considerando el objetivo del artículo se profundizará en las prácticas cotidianas condicionadas y en las relaciones con los otros.

Imagen 1: Categorías emergentes desde los discursos de trabajadores y trabajadoras.

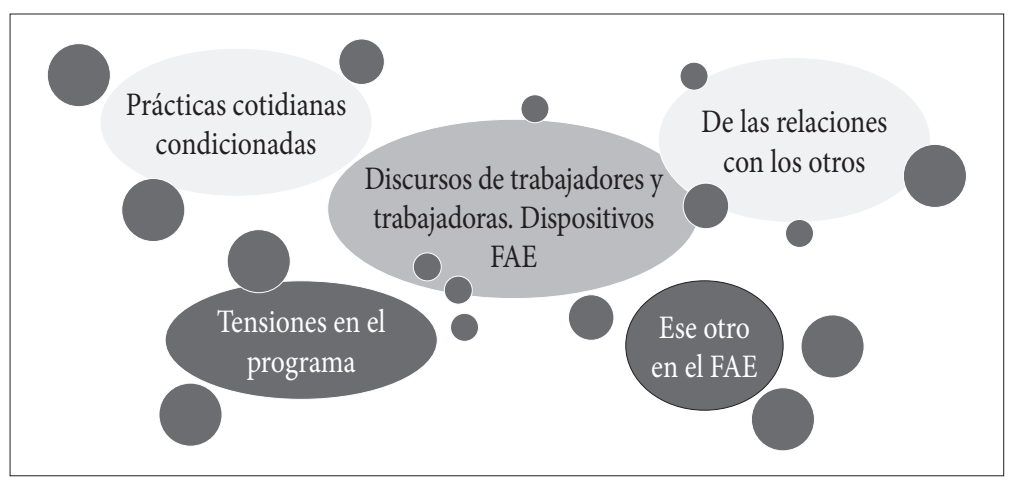

Fuente: elaboración propia. 


\section{De las prácticas cotidianas condicionadas por lo técnico administrativo en el Programa Familia de Acogida Especializada}

La primera categoría que surge desde los discursos de los y las trabajadoras del dispositivo FAE, son las prácticas cotidianas condicionadas, como representaciones que tienen de sí mismos y de las condiciones que los determinan; más bien lo que hacen y la manera en que lo hacen; más precisamente aún, de las formas de racionalidad que organizan las maneras de hacer. Por lo tanto fueron comprendidas como acciones que se producen en el dispositivo desde los mecanismos normativos, técnico y administrativos, como, por ejemplo:

(...) las nuevas orientaciones los plazos están súper acotados, por ejemplo, ingresa un niño ese niño tiene desde ya dos meses plazo para hacer un diagnóstico, al niño, a la familia de origen y a la familia de acogida y tener ese informe listo para el Tribunal. (E3)

Un aspecto tiene que ver con las prácticas moldeadas por los marcos regulatorios, al momento de tener que responder los requerimientos de los lineamientos y el desempeño de las y los trabajadoras/es, siguiendo la normativa regulatoria institucional y también el funcionamiento del programa, determinado por la institucionalidad tanto de SENAME como de la organización colaboradora que ejecuta el programa FAE. Además, las orientaciones técnicas se han ido actualizando, con ello los plazos de intervención se han acotado, de esta manera el Tribunal de Familia, como dispositivo, también regula la intervención y las prácticas cotidianas. A este respecto una entrevistada plantea:

(...) Nuestro trabajo tiene que ver con las habilidades parentales y la mantención de un niño en una casa, eso po' trabajar el caso, en las tres áreas individual, familiar y comunitario principalmente familiar. (E4)

Las prácticas están reguladas por marcos conceptuales determinados; un ejemplo de ello son las habilidades parentales, mode- 
lando el actuar de las familias y de los NNA. Este modelamiento es principalmente cognitivo-conductual, con la apuesta de "reproducir roles socialmente aceptados", además de modelar para lo que la normativa considera positivo.

Por otra parte, los discursos de los y las trabajadoras dan cuenta que sus prácticas están organizadas y determinadas por un funcionamiento interno propio de su quehacer, de acuerdo con los mecanismos técnicos, y se encuentran condicionadas por el marco normativo del programa. Además, se suman prácticas de:

(...) Evaluación de las familias de acogidas externas, habilitación o evaluación de idoneidad de familias acogidas extensas, los tíos los primos los hermanos mayores, los abuelos, evaluación del estado general del ingreso del niño. (E3)

Propio del quehacer de los trabajadores es realizar evaluaciones permanentes a las familias de origen, de acogida, externas y extensas y a la situación de NNA. Esta práctica evaluativa permanente es "el centro de los procedimientos que constituyen al individuo como objeto para un conocimiento y efecto de poder" (Focault, 1987, p.194). Otra táctica regulatoria que se reconoce como práctica tiene que ver con que:

(...) Se realizan visitas domiciliarias, para resguardar el bienestar de los niños, niñas adolescentes, se realizan informes de avances que se entregan a los tribunales, donde uno da a conocer la situación actual y proteccional de los niños. (E8)

La visita domiciliaria, como técnica de biopoder, se reconoce como estrategia evaluativa y de control, y determinada por otra institución para dar cuenta de la situación. La visita domiciliaria se establece como un mecanismo de resguardo, incluso de protección y de producción de información, se invisibiliza como táctica de regulación del comportamiento de los sujetos.

Otro aspecto que configura la categoría "practicas condicionadas", tiene relación con la subcategoría: enfoques de intervención, que aborda la intervención realizada en función de los objetivos 
determinados. Un programa debe "establecer una red de formas mensurables que aclaren en qué sentido y a través de qué modelos operacionalizables de diseño, gestión y evaluación, esto se llevará a cabo" (Matus, 2009, p. 243). Pero estos están determinados por el marco regulatorio, así se pudo constatar desde el discurso de los trabajadores y trabajadoras:

El enfoque es sistémico, de todas maneras, aquí tú no puedes trabajar en un área solita, sin considerar todo lo que hay al lado o detrás del niño, aquí el niño es en torno a su familia, en torno a la familia guardadora. (E1)

El enfoque sistémico pasa a ser uno de los referentes que dominan las intervenciones, asumiendo que las familias están constituidas por un conjunto de personas que interactúan entre sí y con su entorno. De acuerdo con estos principios, la familia se entiende fundamentalmente desde este enfoque. Por otra parte, se reconoce como mandato y articulador de la intervención el enfoque de derechos:

Enfoque de derecho y de estos derechos: el principal es el derecho a que los niños y niñas permanezcan dentro de una familia, desde ahí se trabaja. (E 3)

Los entrevistados se reconocen como responsables de garantizar el respeto, la protección y el cumplimiento de los derechos de NNA, lo que les permitiría analizar las desigualdades que enfrentan y corregir las prácticas discriminatorias mecanizadas que obstaculizan el desarrollo de las familias.

\section{De las relaciones con los otros}

La segunda categoría que se articuló a través de los discursos de los entrevistados fueron las relaciones que existen dentro de este espacio. A este respecto se advierte que las relaciones entre trabajadores/as, NNA y familias de origen y de acogida en el FAE están "sometidas a hábitos, a reglas, a órdenes, a una autoridad que se ejerce continuamente en torno suyo y sobre él” (Foucault, 2001, p. 121), que las configura y regula. 
Un aspecto de esta dimensión se vincula con la regulación y búsqueda de la normalización de las familias de origen, la que llega a través de una resolución judicial, determinada por un juez o jueza del tribunal de familia. Las familias 'ingresan' subordinadas a la resolución, pero hay otras que se resisten a esta regulación, son investidas como "familias que no adhieren al programa", o "disfuncionales al sistema”. Los profesionales regulan el accionar de las familias, ya que éstas deben firmar un consentimiento; por lo tanto, no implicaría la posibilidad de resistencia.

(...) mira la niña espera hace cuatro años y medio y tiene esa misma edad o sea que desde que ella nació que está internada, entonces no te podemos esperar más: o te ponís las pilas ahora o las condiciones se adecuan a su situación, o simplemente su hija se va a ir en adopción. (E10)

Si las familias "funcionan bien”, marcha la relación, pero ¿qué implica marchar bien?, que las familias se adapten a las competencias parentales establecidas, para que niños y niñas vuelvan, o que se generen una relación de docilidad frente a los y las trabajadoras. Por otra parte:

(...) al principio no es así, yo al principio odiaba a todos y ni siquiera las citábamos ¿cachai? Ahora las citamos, pero te engrupen, eso sí, si en seis meses yo no veo un cambio ¡chao! No trabajo más con ellos. (E4)

Se aprecia un rechazo en la intervención con las familias de origen, un estigma. Se asume una relación personal con las familias, se pone en juego la concepción de "adherencia al programa" esa idea de que cada familia debe responder dócilmente a cada planteamiento en el dispositivo. En el caso que se vea resistencia, o no se vea un cambio significativo, señalan que no siguen el proceso de intervención.

(...) Ellos vienen con el sentimiento de que "les quitaron a los niños", la postura de ellos es mucho más a la defensiva. (E3) 
Existe concordancia respecto de la mirada que tienen las familias de origen al ingresar al programa, donde se manifiesta que estos llegan con la sensación de que "les quitaron a sus hijos" y con una postura defensiva, resistente, con desconfianza. Las familias se sienten presionadas, visualizan inmediatamente el control al cual serán sometidos y obligados.

Otro aspecto de esta categoría dice relación con "los sujetos, pero más objetos" al abordar la cuestión de la vinculación con niños, niñas y adolescentes en el dispositivo de intervención.

(...) Con los niños una bonita relación, te insisto, sobre todo con los que son más pequeños, las intervenciones tienden a ser súper lúdicas. (E 5)

(...) es una relación bien bonita y cercana la que tenemos todos en general con los chiquillos, los más grandes son los porfiados de repente... no nos hacen caso. (E 2)

Se manifiesta que algunos NNA "no obedecen" a los profesionales, expresando una resistencia que es percibida como negativa, configurando la obediencia como un valor en la intervención. Lo que invisibiliza y, por tanto, obstaculiza la comprensión de los NNA y sus singularidades. Ello está acompañado con el discurso de los derechos. Reconocer a los NNA como sujetos está determinado por un marco jurídico y social, pero ¿cómo se aprecia esto en el discurso de los y las trabajadoras en sus acciones?

(...) Si llego a la casa y encuentro que el niño está siendo vulnerado o maltratado, yo tengo la facultad de tomar al niño y llevármelo y sacarlo inmediatamente de la casa, con carabineros y con todo. (E9)

Es un cuerpo-objeto que es tomado y manipulado sin una reflexión más profunda sobre las relaciones de sujeción. Señalar: tenemos que "tomarlos", "ponerlos" y "sacarlos", da cuenta más bien de una posición de objeto que de sujeto, y muestra que quienes intervienen se sienten con la facultad y el poder de tomar decisiones 
y realizar acciones que involucran directamente a los NNA, en lo cual los pueden "sacar" de un hogar y "poner" en otro, sin considerar su palabra, sus sensaciones. Ello pone en tensión el enfoque de derechos en el que se sustentaría la intervención.

Otra subcategoría es la "imagen que configuran otros" en las relaciones con los entrevistados. Es una representación vinculada a su acción:

(...) Somos mal vistos, porque somos los que quitamos a los niños, porque tomamos decisiones, los hogares y el FAE son los que se quedan con ellos... y nosotros los sacamos de sus hogares. (E10)

(...) nosotros les vamos a quitar al niño, piensan que nosotros somos la mano negra de SENAME. (E8)

Se perciben como responsables de desarraigar a niños y niñas de sus familias y, por tanto, la relación de poder sí se admite, más aún cuando ellos mismos reconocen que son quienes los desarraigan y no el articulado institucional.

Ser "la mano negra del SENAME", da cuenta de cómo sus relaciones son significadas, como reproducen la normativa, los lineamientos y marco regulatorio institucional. Ellos son los que directamente ejercen el control de las familias y los niños, niñas y adolescentes durante el proceso de intervención.

\section{Dimensiones sustantivas del dispositivo}

Un segundo nivel de resultados se relaciona con un movimiento deductivo, realizado a partir de ciertas dimensiones de la teoría sustantiva. A continuación, se presentan algunos hallazgos al respecto.

\section{Las configuraciones de sujeto en el dispositivo}

Desde los discursos de los y las trabajadores/as del dispositivo FAE emergen distintas configuraciones de sujeto, que proporcionan versiones diversas de lo que representan los individuos en este 
dispositivo de intervención social: un sujeto controlado y/o sujeto "sujetado".

Se aprecia a los NNA, sus familias de origen y las familias de acogida como entidades estandarizadas que se definen en forma permanente como vulneradas, protegidas, institucionalizadas, objetos de intervención. Se trata de una construcción discursiva que establece "entre los seres naturales, un sistema de identidades y el orden de las diferencias" (Foucault, 1968, p. 137). Esto categoriza a los individuos que son instalados dentro de este dispositivo.

Se trata de una configuración de sujeto institucionalizado, inserto en una red, bajo medidas de protección, que, a la vez, es objeto de intervención en el dispositivo, sobre quien se establecen medidas que buscan normalizar su situación o condición, bajo mecanismos de control sociojurídico, es decir, de un "aparato de observación, de registro y de encauzamiento de la conducta" (Foucault, 2001, p. 161). Es un sujeto - objeto de intervención de las decisiones institucionales bajo los mecanismos de control y normalización que establece el Estado. Esto se ejemplifica a continuación:

(...) nosotros informamos, de cómo los niños van en sus procesos, nosotros vamos evaluando si el niño vuelve o no a su familia de origen. (E6)

Desde esta idea de objeto emerge la figura de sujeto sujetado a la institucionalidad, sujeto que debe obedecer a la tendencia homogeneizadora de instituciones, como los tribunales, el SENAME, entre otras. Con ello se identifica "por un lado, poder legislador y por el otro, sujeto obediente" (Foucault, 1991, p. 51). Esta configuración se hace extensiva a las familias que se encuentran vinculadas a la intervención del programa, familias de origen y familias de acogida, ya que desde el dispositivo se "controla y vigila todas y cada una de las conductas” (Apreda, 2003, p. 13).

los vigilamos y no los dejamos tomar decisiones, es como eso que te dicen: que a usted tengo que contarle todo. Eso, como a ellos les quita libertad. (E10) 
Emerge la idea de "sujeto controlador", que "impone la mejor relación entre un gesto y la actitud global del cuerpo, que es su condición de eficacia y de rapidez... Un cuerpo bien disciplinado forma el contexto operatorio del menor gesto" (Foucault, 2001, p. 140). Por ello, algunos trabajadores, dentro de este dispositivo, se perciben a partir de lo que otros piensan acerca de su rol, a saber, un sujeto que controla las vidas de los niños, las niñas, los adolescentes y las familias, esperando que cumplan con los requerimientos que se imponen desde el dispositivo: normalización y orden.

\section{Mecanismos regulatorios del dispositivo Familia de Acogida Especializada}

Los mecanismos regulatorios del programa FAE, como dimensión, permitieron reconocer a partir de los discursos, de los procesos de subjetivación, de las formas de reproducción de saber y de las formas de poder, cuáles eran sus articulaciones con el proceso gubernamental que se instala jurídica y normativamente sobre los cuerpos de los sujetos que se relacionan con el dispositivo. Esto se evidencia en los discursos que organizan las acciones.

Se puede apreciar la subjetivación que el sujeto hace de la experiencia de sí mismo, pero esa experiencia no es igual para todos, es la experiencia del particular mundo en el que se vive en el dispositivo FAE. Este proceso reenvía a la idea del "sujeto controlador" y del "sujeto sujetado". Los individuos que circulan en el programa van construyendo formas de subjetividad, incididas por mecanismos regulatorios y normalizadores. Además, son formas de objetivación de los modos en que el sujeto ha sido objeto de saber y de poder, para sí mismo y por, sobre todo, sobre un otro.

Por otra parte, la relación de poder/saber es soporte en la subjetivación de los NNA sujetados en el FAE. Ello se refleja en las posiciones de saber que detentan las figuras que se relacionan a partir del control, en tanto profesionales y expertos: un saber único que construye al otro como alguien que "no sabe comportarse", "que no cuida", que "vulnera", "que no cumple", "que es problemático", entre otros. 
(...) nosotros tenemos los conocimientos y la preparación para actuar técnicamente, las familias que vulneran las reconocemos y las denunciamos. (E9)

Ese orden del saber, como lo plantea Foucault (1994), tiene relación con una forma discursiva de control de la producción de nuevos discursos, y en relación directa con el poder, entendiéndolo en este caso como el conjunto de técnicas en virtud de las cuales los sistemas tienen por objetivo y resultado la singularización de los individuos. Este saber, sostenido desde una ideología dominante, de contenido técnico, institucional, proteccional, sociojurídico, puede ser también, por momentos, un poder benevolente.

\section{Las relaciones de poder en el dispositivo Familia de Acogida Especializada}

Esta dimensión es fundamental para comprender el FAE. Cabe destacar que las relaciones se articulan a partir de los distintos sujetos que interactúan en el dispositivo, desde donde emergen vínculos profesionales e institucionales entre y con SENAME, los tribunales de familia, los sujetos del programa, específicamente los NNA, familias de origen, familia de acogida y los trabajadores.

(...) Somos mal vistos, porque somos los que quitamos a los niños, porque tomamos decisiones, los hogares y el FAE son los que se quedan con ellos... y nosotros los sacamos de sus hogares. (E 4)

La relación de poder de refleja en los cuerpos de NNA y sus familias, los que son sometidos a mecanismos de normalización, entendidos como "los modos de acción que no actúan directa e inmediatamente sobre los otros, sino sobre sus acciones" (Foucault, 1994, p. 230).

Cabe destacar que en las prácticas cotidianas que se desarrollan en las relaciones entre los dispositivos (FAE con Servicio Nacional de Menores y los tribunales de familia, centros de salud, escuelas, municipios, entre otros), se puede determinar cierta regula- 
ción desde la posición de cada institución, desde donde los trabajadores son sometidos "a reglas, a órdenes, a una autoridad que se ejerce continuamente en torno suyo y sobre él” (Foucault, 2001, p. 121). Esto se grafica en la siguiente cita,

(...) La principal relación es con la unidad de protección, donde están nuestros supervisores técnicos, que son los que visualizan los cumplimientos técnicos de intervenciones a nivel psicológico y a nivel social, y la coherencia interna que se realiza en el plan de intervención, además de las solicitudes entregadas por el tribunal. (E3)

Esto reproduce las relaciones de regulación ejercidas por la institucionalidad, bajo pretexto de velar por la protección de los derechos de NNA. Es así que opera el mecanismo y las relaciones de control sobre la vida de ellos y ellas, siendo regulados también por los tribunales de familia, quien determina las medidas de protección.

En general, las y los trabajadoras/es regulan, controlan, condicionan y disciplinan la vida de los sujetos, llámese NNA, familia de origen y/o familia de acogida. Así se disciplina, se "fabrican así cuerpos sometidos y ejercitados, llamados cuerpos dóciles" (Foucault, 1976, p. 126).

Esto refuerza la idea ya expresada de que las relaciones de poder producen en el FAE cuerpos sometidos y normalizados por las orientaciones técnicas establecidas por el marco normativo y por los dispositivos de SENAME, que sitúan a los sujetos en posiciones subordinadas en la medida en que éstos deben adaptarse a sus requerimientos. Así, las "relaciones de poder operan sobre una presa inmediata; lo cercan, lo marcan, lo doman, lo someten a suplicio, lo fuerzan a unos trabajos, lo obligan a unas ceremonias, exigen de él unos signos" (Foucault, 2001, p. 99).

Es así como el dispositivo FAE se encuentra en constante movimiento e interacción, tanto entre los sujetos internamente (trabajadoras/es, NNA, familias de origen y familias de acogida), como 
con la institucionalidad externamente (SENAME, tribunal de familia, centros de salud, establecimientos educacionales y programas colaboradores).

\section{Rearticulación del dispositivo FAE}

En este apartado se presentarán algunos resultados de la investigación, producto de las vinculaciones inductivo-deductivas que se realizaron en el proceso de interpretación. Esto con base en las categorías que se construyeron y de las subcategorías que emergieron desde las prácticas discursivas de las y los trabajadoras/ es del dispositivo de investigación: Familia de Acogida Especializada. Estas fueron vinculadas con las dimensiones sustantivas y pondrán en evidencia las relaciones y las tensiones que permiten comprender el FAE como dispositivo de intervención social.

\section{El dispositivo FAE: desde las relaciones evidenciadas a la investidura del sujeto}

Teniendo en consideración las relaciones que se originan en el dispositivo FAE, se advierte que éstas configuran a los sujetos con ciertas investiduras ideológicamente dominantes. Ello da cuenta, como se indicó, de un sujeto-sujetado que debe obedecer a las reglas, requerimientos y normatividad establecidas en los marcos y mecanismos regulatorios de los dispositivos. Esto implica que las regulaciones normativas son estructurantes de un perfil de sujeto.

Dicha configuración alude también a las familias que forman parte de este dispositivo, las que se ven sometidas a las regulaciones de las y los trabajadoras/es, particularmente de profesionales del dispositivo, quienes sostienen su actuar en los marcos normativos de SENAME y en mecanismos legales. Así se configura el sujetocontrolador, que encuentra sus fundamentos en los mecanismos referidos, pero también en concepciones paradigmáticas que invisten a los sujetos. De esta manera, se reproduce una lógica de dominación sobre ellos y ellas, siendo el dispositivo FAE parte de una red que reproduce la regulación, haciendo emerger resisten- 
cias y tensiones, pero que son coercitivamente reguladas con la presión de otros dispositivos, como los tribunales de familia.

\section{Dispositivo FAE como espacio de control y normalización desde prácticas de trabajadores/as articulados/as por mecanismos regulatorios}

En relación con las prácticas evidenciadas desde los discursos de los y las trabajadoras, se aprecia un tipo de subjetivación dominada por los marcos regulatorios, que configura la institucionalidad y los marcos normativos, llámense orientaciones técnicas, leyes de protección, normativas, perspectivas teóricas, profesionales y personales en ellos y ellas. Hay una subjetivación sujetada a estos mecanismos, evidentemente naturalizada; en tal sentido, no hubo críticas o alusión a prácticas de resistencia, no se aprecian campos de posibilidad o puntos de fuga.

Los marcos regulatorios del dispositivo, administrados por SENAME y/o tribunales, controlan y orientan las prácticas de los y las entrevistadas en el dispositivo FAE, pero a la vez vigilan y someten los cuerpos de niños, niñas, adolescentes, como también de sus familias, reproduciendo lógicas y requerimientos que los marcos reguladores dominantes. Desde el marco regulatorio surge una serie de requerimientos técnicos, a los cuales los trabajadores deben responder; estos poseen directa relación con sus prácticas, centradas en normalizar al sujeto, parte del objetivo de la intervención, siguiendo de manera estructurada los lineamientos técnicos señalados en el marco normativo.

Como se mencionó, los sujetos que se encuentran inmersos en este dispositivo son controlados, debiendo adaptar sus comportamientos a las orientaciones técnicas de cada marco regulador y en las que prevalece la figura del SENAME, el Tribunal de Familia y las redes con las cuales interactúan, tales como establecimientos educacionales, centro de salud, y programas colaboradores de la red SENAME. 


\section{Dispositivo FAE: subjetivaciones desde las relaciones de poder/saber}

Las nociones que emergieron de los discursos de las y los trabajadoras/es evidencian determinadas formas de subjetivación por parte de quienes se encuentran en el dispositivo FAE. Se deduce que las relaciones de poder operan sobre un cuerpo específico, cuerpos sometidos, controlados y regulados por quienes ponen en práctica los marcos regulatorios del dispositivo; pero además subjetividades investidas y coaccionadas por categorías tales como "NNA institucionalizado/a". Esto ocurre en toda la red de dispositivos que administra SENAME, naturalizándose esta trayectoria sin problematizarla, sin generar una crítica que la ponga en cuestión o se resista a ella.

Por otra parte, las relaciones de poder se ejercen sobre las familias de origen y de acogida, quienes deben responder a una serie de requerimientos técnicos, provenientes de marcos normativos y orientaciones que permiten a los profesionales el ejercicio de un poder en forma directa. Son ellos quienes regulan, controlan, condicionan y disciplinan la vida de cada sujeto, por medio de los requerimientos de SENAME y el Tribunal de Familia. El tener que trabajar con la familia vulneradora es la propuesta a la que se resisten algunos trabajadores, reconociendo una relación basada en la coacción de sus prácticas y decisiones.

Con respecto al funcionamiento de los dispositivos, se advierte que el FAE es una red de relaciones de poder y configuraciones de sujetos sometidos a mecanismos que actúan sobre cuerpos, sobre sus trayectorias de vida, que los vuelven dóciles en tanto objeto institucionalizado de intervención, cuerpo disciplinado y regulado por el gobierno del Estado. Gobernar a través de este dispositivo implica estructurar el posible campo de acción de los otros. El efecto de relacionamiento propio del poder se encuentra en el área de modos de acción singulares que son el gobierno, o sea gobernar al otro.

Así, el poder gubernamental, conducido por vía de dispositivos como el FAE, logra disponer de las vidas y cuerpos de quienes se 
relacionan en esa red, a través de mecanismos y tácticas de sometimiento. "Y esas tácticas se aplican ya no para mandar sobre un determinado territorio, sino para gobernar a los hombres y a las cosas o, más precisamente, a las interacciones que se producen entre esos dos conjuntos" (Foucault, 2006, p. 376).

\section{Apreciaciones finales}

Lo planteado hasta aquí muestra la manera en que la dimensión política se manifiesta en los dispositivos de intervención social. Ello se ve reflejado en procesos desplegados a partir de un cierto orden o una cierta racionalidad, que se consolidan y mantienen desde las relaciones de poder que se expresan en la micropolítica presente en los programas FAE.

Los mecanismos políticos característicos del liberalismo circulan por los programas FAE, quedando en evidencia, desde el discurso de los y las trabajadoras, la reproducción de modos de existencia dentro de los cuales las subjetividades funcionan por (auto) regulación. Estas tecnologías buscan que los y las gobernadas hagan coincidir sus deseos, esperanzas, decisiones, necesidades y estilos de vida con objetivos gubernamentales fijados de antemano por los marcos reguladores administrados por la institucionalidad, llámese SENAME, Tribunal de Familia u otro.

La investigación evidencia las regulaciones de los cuerpos y subjetividades de los sujetos en el dispositivo FAE y reconoce la existencia de relaciones biopolitizadas a través de mecanismos específicos, los que se manifiestan directamente mediante la normativa establecida a nivel "proteccional" de la niñez. Esto se logra evidenciar en el discurso de las y los profesionales, quienes regulan la vida de los sujetos mediante la normalización e individuación reflejadas en sus prácticas y normativas técnicas que permean su cotidianidad. Así, el FAE se establece como la red de relaciones que implica una tecnología regulatoria por sobre todo de los cuerpos de NNA. Estas relaciones de poder/saber múltiples han construido durante décadas un cuerpo social de la niñez, cosificada, suje- 
tada; y estas relaciones, que han estado bajo el sometimiento de la "protección del gobierno neoliberal" no pueden disociarse, ni establecerse, ni funcionar sin una producción, una acumulación, una circulación, un funcionamiento del discurso que es reproducido por los y las trabajadores/as del FAE. Con ello, las funciones que reproducen de estas técnicas y procedimientos son: vigilar, controlar, normalizar, creando así una individualidad orgánica.

Podemos concluir que en el dispositivo de intervención FAE es la norma (orientación técnica, ley de protección, ley de menores, ley de tribunales de familia, ley de subvenciones) la que circula y articula lo disciplinario y lo regulador; permite cotidianamente controlar el orden disciplinario del cuerpo, tanto de NNA, de las familias de origen y las familias de acogida, inclusive a los propios trabajadores y trabajadoras. De esta manera, la gubernamentalidad liberal chilena se configura en ese movimiento articulado entre disciplina y regulación, en cuanto a prácticas sistemáticas de sujeción de NNA y sus familias, por medio de mecanismos de poder que reclaman para sí una verdad y que son mediados preferentemente por profesionales. Estos últimos se arrogan el derecho de interrogar a la verdad sobre sus efectos de poder $\mathrm{y}$ al poder sobre sus discursos de verdad.

La crítica sería el proyecto de una in-servidumbre voluntaria y racional, la búsqueda de una resistencia que debemos provocar, una indocilidad reflexiva-activa. Esto implicaría una acción política de des-sujeción en el juego cotidiano de los dispositivos, y que Foucault (1995) llamó: "política de la verdad".

\section{Bibliografía}

Agamben, G. (2002), Homo Sacer I. El poder soberano y la nuda vida. Madrid, España: Editora Nacional.

Agamben, G. (2015). ¿Qué es un dispositivo? Buenos Aires, Argentina: Anagrama.

Aguilar, G. (2008). El principio del Interés Superior del Niño y la Corte Interamericana de Derechos Humanos. Estudios Constitucionales, Centro de Estudios Constitucionales de Chile, (1), 223-247. 
Apreda, G. (2010). La Concepción de Sujeto en Michel Foucault. Revista Borromeo, (1), 5-30.

Bresser, L. y Cunill, N. (1998). Lo público no estatal en la reforma del Estado. Buenos Aires, Argentina: Paidós.

Botticelli, S. (2015). La Gubernamentalidad del Estado en Foucault: Un Problema Moderno. Praxis Filosófica Nueva Serie, (42), 83-106.

Butler, J. (2008). Cuerpos que importan. Sobre los límites materiales y discursivos del sexo. Buenos Aires, Argentina: Paidós.

De La Maza, G. (2004). Políticas públicas y sociedad civil en chile: el caso de las políticas sociales (1990-2004). Revista Política, (43), 105-148.

De Souza Minayo, M. (2010). Los conceptos estructurantes de la investigación cualitativa. Revista salud colectiva, (6), 251-261.

Filgueira, C. (2000). Bienestar y ciudadanía: viejas y nuevas vulnerabilidades, en V. Tokman y G. O’Donnell (comps.), Pobreza y desigualdad en América Latina (pp. 123-147). Buenos Aires, Argentina: Paidós.

Foucault, M. (1968). Las Palabras y las Cosas. Buenos Aires: Siglo XXI.

Foucault, M. (1979). Microfísica del Poder. Madrid, España: La Piqueta.

Foucault, M. (1991). Historia de la Sexualidad I, La Voluntad de Saber. Madrid, España: Siglo XXI.

Foucault, M. (1994). La hermenéutica del Sujeto. Madrid, España: Ediciones de la Piqueta.

Foucault, M. (1999). Estrategias de Poder. Barcelona, España: Paidós.

Foucault, M. (2000). Defender la Sociedad. Buenos Aires, Argentina: Fondo de Cultura Económica.

Foucault, M. (2001). Vigilar y Castigar. Buenos Aires, Argentina: Siglo XXI.

Foucault, M. (2007). El nacimiento de la biopolítica. Buenos Aires, Argentina: Fondo Cultura Económica.

Gadamer, H-G. (1993). Verdad y método Fundamentos de una hermenéutica filosófica. Salamanca, España: Ediciones Sígueme.

Gruninger, S. (2003). Las ONGs durante la transición chilena: Un análisis de su respuesta ideológica frente a su incorporación en políticas sociales de índole neoliberal. Revista MAD. Departamento de Antropología. Universidad de Chile, (9). Recuperado de http://rehue.csociales.uchile.cl/publicaciones/mad/09/paper06.pdf 
Martínez, V. (2011). Análisis evaluativo del programa familia de acogida especializada de Sename. Recuperado de http://www.programassociales.cl/pdf/otras_eval/2015/EV60914_1.pdf

Matus, T., Haz, A., Razeto, A., Funk, R., Roa, K. y Canales, L. (2008). Innovar en calidad: Construcción de un modelo de certificación de calidad para programas sociales, en Camino al Bicentenario Propuestas para Chile (pp. 227-270). Santiago, Chile: Pontificia Universidad Católica de Chile, Gobierno de Chile, Cámara de Diputados y Biblioteca del Congreso Nacional de Chile.

Oyarzún, A., Dávila, O. y Hatibovic, F. (2008) ¿Enfoque de derechos o enfoque de necesidades? Modelo de gestión para el desarrollo de un sistema local de protección de derechos de la infancia y adolescencia. Santiago, Chile: SENAME y Ediciones CIDPA.

Ovejero, A. y Pastor, M. (2001). La dialéctica saber/poder en Michel Foucault: Un instrumento de reflexión crítica sobre la escuela. Aula abierta, (77), 99-107.

Rojas, J. (2010). Historia de la Infancia en el Chile Republicano 18102010. Santiago, Chile: Ocho Libros.

Salazar, G. (2003). Labradores, peones y proletarios. Formación y crisis de la sociedad popular, Siglo XIX. Santiago, Chile: LOM Ediciones.

Salazar, G. (2006). Ser niño huacho en la historia de Chile. Santiago, Chile: LOM Ediciones.

Sánchez, S. y Villarroel, R. (2017). Tensiones en la intervención social: (des)encuentros en la relación Estado-Ong. Estudio de caso sobre Ong que opera la política social de infancia. Revista Trabajo Social, (91), 3-16.

Sautu, R. (2005). Manual de Metodología. Construcción del marco teórico, formulación de objetivos y elección metodológica. Buenos Aires, Argentina: CLACSO.

Sename. (2010). Catastro de la Oferta Programática de la red Sename. Recuperado de http://www.sename.cl/wsename/otros/op/ CATASTRO-201012.pdf

Sename. (2015). Línea de acción programas. Orientación técnica Programa familias de acogida especializada, programa de protección especializado Fae/pro. Recuperado de http://www. sename.cl/wsename/licitaciones/p7_03-06-2015/Orientaciones_Tecnicas_FAE-PRO.pdf

Sename. (2015). Anuario estadístico. Datos del Sistema de Registro de Información Estadística SENAINFO. Recuperado de http:// 
Dispositivo de intervención y gubernamentalidad del sistema proteccional de la infancia /Cortés

www.sename.cl/wsename/images/anuario_2015_final_200616.pdf

Torres, O. (2008). Niñez, políticas públicas y sociedad civil. Revista $M A D,(3), 17-45$. 